\title{
Concentric Layout, a new scientific data distribution scheme in Hadoop file system
}

\author{
Lu Cheng, Pengju Shang, Saba Sehrish, Grant Mackey, Jun Wang \\ University of Central Florida \\ lucheng@knights.ucf.edu
}

\begin{abstract}
The data generated by scientific simulation, sensor, monitor or optical telescope has increased with dramatic speed. In order to analyze the raw data fast and space efficiently, data pre-process operation is needed to achieve better performance in data analysis phase. Current research shows an increasing tread of adopting MapReduce framework for large scale data processing. However, the data access patterns which generally applied to scientific data set are not supported by current MapReduce framework directly. The gap between the requirement from analytics application and the property of MapReduce framework motivates us to provide support for these data access patterns in MapReduce framework. In our work, we studied the data access patterns in matrix files and proposed a new concentric data layout solution to facilitate matrix data access and analysis in MapReduce framework. Concentric data layout is a hierarchical data layout which maintains the dimensional property in large data sets. Contrary to the continuous data layout adopted in current Hadoop framework, concentric data layout stores the data from the same sub-matrix into one chunk, and then stores chunks symmetrically in a higher level. This matches well with the matrix like computation. The concentric data layout preprocesses the data beforehand, and optimizes the afterward run of MapReduce application. The experiments show that the concentric data layout improves the overall performance, reduces the execution time by about 38\% when reading a 64 GB file. It also mitigates the unused data read overhead and increases the useful data efficiency by $32 \%$ on average.
\end{abstract}

\section{Introduction}

In these days, more and more scientific applications have been benefiting from the MapReduce framework[9]. These applications share the property that they generate, collect and maintain vast volumes of data, and also require large computing resource to process data[7]. For example, earthquake prediction and analytic model collect up-dated and detailed data of earth activity around the world[8] to let geologists generate a more accurate and efficient earthquake analytic model. These data are collected in every second and delivered to computation unit for process. Many other scientific research applications such as bioinformation model, vision simulation, climate prediction and realistic graphic animation shares same properties generate, store and process multi-TeraByte data. MapReduce is a good candidate for these applications as MapReduce jobs are distributed into multiple sub-jobs and processed concurrently. The distributed property improves the processing speed and improves the execution efficiency.

For many analytic applications, data set are generated and stored in a matrix manner naturally. For example, the weather monitors application sensing and records the temperature and humidity variation in real time. Scientists analyze posted data to forecast the future weather changes. One impelling analytic requirement is to compare the data values among different periods in the same day or the same time among different days. Apparently, storing the data set into a matrix manner will bring performance benefit for the future analysis. Instead of reading the entire data set, the scientist just needs to read the data set in the same row to analyze the temperature change during the same day or to review the data set in the same column to analyze the humidity variation in a month. Therefore, the way the dataset is stored in a file system has an intimate relationship with how it is accessed.

In distributed file systems like HDFS (Hadoop Distributed File System)[6] which adopts MapReduce framework, the data is stored sequentially and read stream in default. Unfortunately, such storage feature breaks the aforementioned intimate relationship between how the dataset is stored in HDFS and the way data analysis program accesses. Using the weather 
monitoring application as an example, when file is stored in HDFS sequentially, the data in the same column are separated and distributed among the entire file system. When one column of data are needed, instead of just reading one column, the whole file will be accessed. An inappropriate data layout will affect the data processing efficiency as it results in reading excess amount of data than actually being required. Meanwhile, storing the data set in a file system with one access pattern cannot fit various applications with different access patterns. After the monitor data set is generated and stored in a file system, the analytic applications with various access patterns will access the data set to perform different data analyses. For example, temperature data is used for analyzing data fluctuations in different time periods, like in a day or in a year. Based on the specific analytic requirement, the data set will be accessed in either row based or in column based.

In order to deal with the aforementioned challenges, we propose a new concentric data layout scheme. Concentric data layout is a hierarchical data layout algorithm which stores data in a two-dimensional way. Its unique combination of row based access pattern and column based access pattern makes it works well for many scientific applications using matrix data structures. In concentric data layout, affiliated data are stored into the same chunk and hence maintain the original logical properties. As the data are stored in two dimensional manners, accessing the data in either row or column will lead to comparable performance, and realize the optimal overall performance when applications access the same matrix data set in different patterns. Our experiments show that the concentric data layout is able to significantly improve the I/O performance by reducing the total number of chunks being accessed for scientific analytic applications using matrix data structures.

The paper is organized as follows, section 2 introduces the background of MapReduce framework and matrix related data access pattern. In section 3 , we propose the concentric data layout in detail and discuss the experimental results in section 4. Section 5 introduces the related work while the conclusion and further works are discussed in section 6 .

\section{Background}

In this section, we introduce the HDFS, MapReduce framework and data access patterns in matrix data set in brief.

\subsection{HDFS and MapReduce framework}

The Hadoop Distributed File System (HDFS) is a distributed file system designed to run on commodity hardware[1]. The default storage unit in HDFS is called chunk which usually has the size of $64 \mathrm{MB}$ or $128 \mathrm{MB}$. The aim of HDFS is to benefit the application with large data sets. It provides high throughput access to application data.

MapReduce framework is introduced by Google to support distributed computing with large data sets on cluster of computers[2]. A standard MapReduce program includes two phases, the Map and the Reduce. The input and output for these phases are defined in the form of a key and a value pair. During the MapReduce phases, the MapReduce program will read the contiguous chunks in the Map phase, and generate the $<$ key, value $>$ pair based on the stripes which will be processed by one map task. In the reduce phase, all the stripes assigned to one task will be grouped together. This mechanism works well when data is accessed in a continuous manner which the order of input data does not affect the output result. However, it cannot fit for some analytic applications which require retrieving and processing data complex with particular order and specific manner.

\subsection{Data access pattern in matrix data set}

Continuous Access Pattern: Continuous access pattern is the most widely used data access pattern. In continuous access pattern, data are accessed in roundrobin manner without considering data dependency. It is widely used in applications in which the data are independent with each other; the task can be divided into many sub tasks and processed synchronously. This model fits best with HDFS because the features of streaming access and batch process match with roundrobin access pattern perfectly. The application with round-robin access pattern can yields the best $\mathrm{I} / \mathrm{O}$ performance when processed by MapReduce framework.

Matrix Access Pattern: Row-based or columnbased access pattern are two basic matrix access patterns for matrix data set. It is widely used in scientific analytic applications. For many scientific applications, data are stored with two-dimensional manner in logical file which helps to keep data dependency between each other. However, when the data in logical file with dimension property are stored into physical storage media, data lose their higher level property in file system and become stream bytes. Rowbased data access pattern is similar with continuous data access pattern, but it remains the data relationship with each other. In row-based access pattern, the data closed to each other are located close logically. 


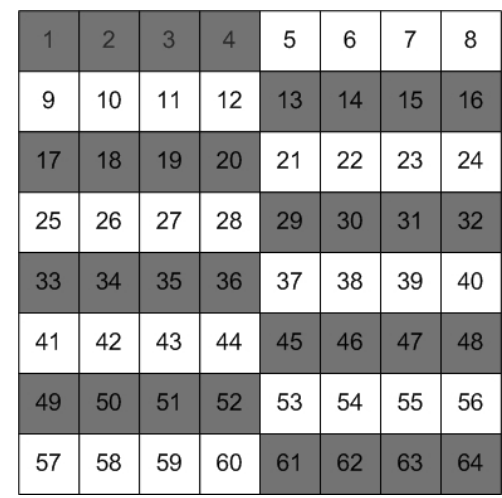

(a) Row-based access pattern

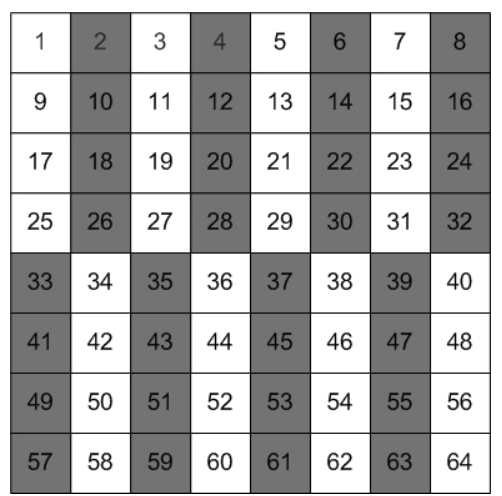

(b) Column-based access pattern

Figure 1. Data access pattern comparison

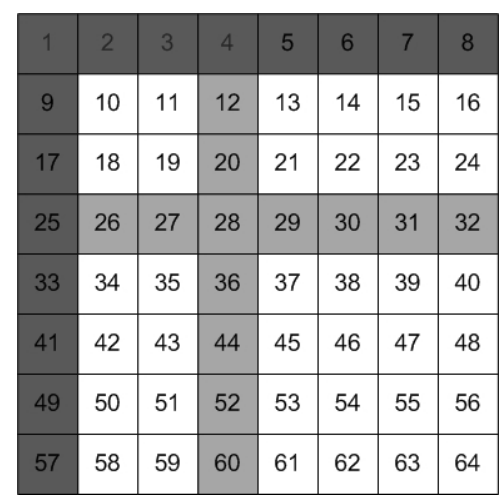

(c) Group based access pattern
Group Access Pattern: Some analytic applications require complex data analysis like group access pattern. Group access pattern is a combined data access pattern which generally used in matrix computation, like matrix multiplication. It requires accessing the row and the column in same matrix set at the same time. The Figure 1(c) demonstrates one example of concentric access that the first row and first column are required. For group access pattern, the data access is two dimensional. It is turned out to be extremely inefficient when data are stored in one dimensional manner. The data utilization rate is decreased because only a small part of accessed data is useful for further analysis.

\section{Concentric Data Layout}

In this section we propose concentric data layout, a matrix-specific data layout optimization strategy to benefit the matrix data access pattern and group data access pattern.

\subsection{Problem Description}

In HDFS, the data are stored continuously and read as stream by default. The problem of this method is that it generates very poor performance for many noncontinuous access patterns. The non-contiguous access pattern generally maps stripes to a distributed set of chunks and results in small I/O problem. The noncontiguous data access impacts the performance in two ways. First, it results in reading excess amount of data than required; second, the stripes assigned to a task may map to a large number of chunks, making the task scheduling extremely challenging.

For example, in practical applications such as matrix operations, accessing column data is very common. However, the default continuous storage data layout results in excessive chunks access with terrible data utilization rate, and arise in extensive data overload. In order to read small data in KBs, the block with $64 \mathrm{MB}$ need to be retrieved. The research indicates that the small $\mathrm{I} / \mathrm{O}$ problem is caused by the fact that the file is treated as linear bytes in the file system, and loses the higher level property at the lower level of file system. For example, from the user's point of view, it is nature to store the data in a multidimensional way in a matrix operation as it is easier to express the data dependency and other information. However, when the file is stored in a linear file system, the multidimensional array is flattened into one dimensional array and the higher level information is lost at the lower level file system, unrelated data is retrieved substantially when user tries to read data with certain relationship. Therefore, in order to improve the reading efficiency for matrix access pattern, new data layout needed to be proposed.

\subsection{Concentric Algorithm}

We propose a data restructuring algorithm for matrix data access pattern and group data access pattern which are common in scientific applications. Concentric data layout is a hierarchy data restructuring strategy that maintains the dimensional property in multiple- dimensional way.

Figure 2 indicates the possible problems which may be aroused by continuous data layout. In figure 2 we show a $8 \times 8$ two dimensional matrix file with a chunk size of 4 elements. Suppose each map task processes one chunk, and chunks are stored consecutively, e.g. chunk 0 contains elements $1,2,3$ and 4 , chunk 1 contains 5, 6, 7 and 8 and so on.

From the Figure 2 we can see this continuous storage method flats the two dimensional matrix into a linear sequence of elements. Each element just maintains the information about its peers within the 


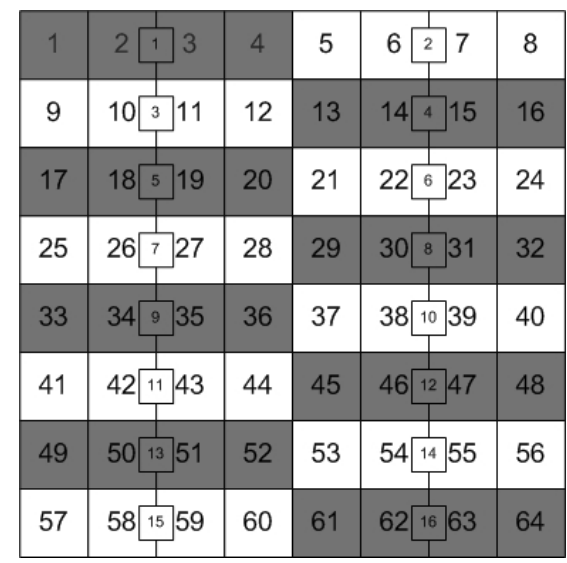

Figure 2. Row based access pattern in matrix data set

same row, but lose the information about the neighbors in the same column. Therefore, this data layout is just suitable for row based access pattern. Assuming the first row of data in the array is needed to be processed, the first row with 2 chunks will be processed by two map tasks. In this case, all the data in accessed chunks are useful and the data access efficiency is $100 \%$. However, when data access pattern turns into vertical, the $\mathrm{I} / \mathrm{O}$ performance becomes disappointing. For example, when the first column is needs to be processed, the whole file, from chunk 0 to 16 will be processed because data are stored sequentially. It greatly deteriorates the I/O performance as the whole file is retrieved, but only the first elements in the chunk with even chunk numbers are useful. In this case the data utilization is only 12 . Considering a matrix file with a size of $64 M \times 64 M$ and the elements with the size of $64 \mathrm{~KB}$., in worst case, each 64MB chunk will be processed with only $64 \mathrm{~KB}$ useful data, leaving the data access efficiency lower near to $0 \%$. The above examples sufficiently show the inflexibility of continuous data layout and demonstrate it cannot be adapted to the requirement of variable access patterns in matrix file.

Contrary to continuous data layout, data are stored in multidimensional way in concentric data layout. The deployment of matrix file can be represented as a $N \times N$ matrix and each data element has a small data size like $1 \mathrm{~KB}$. Meanwhile, the size of each chunk, which is $64 \mathrm{M}$ by default, can be treated as a $k \times k$ submatrix. Therefore, the whole file can be divided into multiple sub-matrixes and each sub-matrix represents a multidimensional chunk. Instead of store the data into the chunk linearly, the concentric data layout stores the data within the same sub-matrix into one chunk.

Compared with the general data layout of linear data storage, the concentric data layout maintains the multi-dimensional property of the matrix. For each

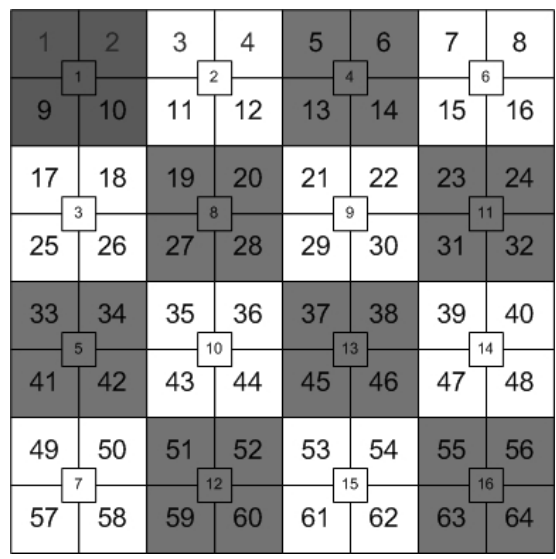

Figure 3. Concentric data layout

chunk, it stores a small square part of the data, the data within the same chunk not only knows its right and left elements (elements in the same row), but also the elements above and below it (elements in the same column). Furthermore, unlike traditional data layout in HDFS where the chunks are stored sequentially in file system, chunks in concentric data layout are stored symmetrically. Starting with the chunk located at the diagonal, chunks in rows and columns are stored into file system alternately. By storing the chunks symmetrically, accessing row and column will retrieve the same number of chunks. The symmetric storage strategy aims to yield the best average I/O performance in group access pattern.

Figure 3 indicates the implementation of concentric data layout in a two dimensional matrix file. It shows that the concentric data layout is a hierarchy data layout which maintains the dimensional property. In our example, the 2-dimensional matrix has the size of $8 \times 8$ and the chunk size is 4 . Therefore, the matrix can be divided into $162 \times 2$ sub-matrixes, each of which contains 4 elements. For example, elements 1, 2, 9 and 10 within the first square belong to chunk 1 , elements 3, 4, 11 and 12 in second square belong to chunk 2, and so on. Chunk 1 at the intersection of row 1 and column 1 is stored into HDFS first, and chunks beside and below are stored symmetrically. Repeat this process until reach the end of a row or column. When reach the end of the row or column, the chunk at the intersection of next row and column start a new cycle.

For matrix in Figure 3, we assume each map task processes one chunk, and then processing whole file requires 16 map tasks in total. When data in the first row is required, chunks 1 to 7 are accessed; the data access efficiency is $28 \%$. Likewise, the same chunks will be accessed with the data access efficiency of $28 \%$ when the data in the first column is needed. Compare to continuous data layout (Figure 1), the number of chunks accessed increased from 2 chunks to 7 chunks 
when reading the first row of the array. However, the number of chunks accessed dropped from 16 chunks to 7 chunks in column data access pattern. Considering the probability of row based or column based access pattern is independent, the average number of accessed chunk is dropped from 9 chunks per access to 7 chunks per access. Concentric data layout achieves better performance with group access pattern, where a row and a column are accessed at same time. Suppose the first row and first column are required by analytic application, 7 chunks are retrieved when running the MapReduce program with concentric data layout. Without concentric data layout, the number of chunks needed to be retrieve is 16 , which means the MapReduce program have to read the whole file to receive all required data. For a $64 M \times 64 M$ matrix file with the chunks size of $8 K \times 8 K, 16 \mathrm{~K}$ chunks are accessed when the first row or column are accessed. Comparing with the continuous data layout with an average access number of 64M chunks per access, the saving is astonishing.

The pseudo code for concentric data layout is as following.

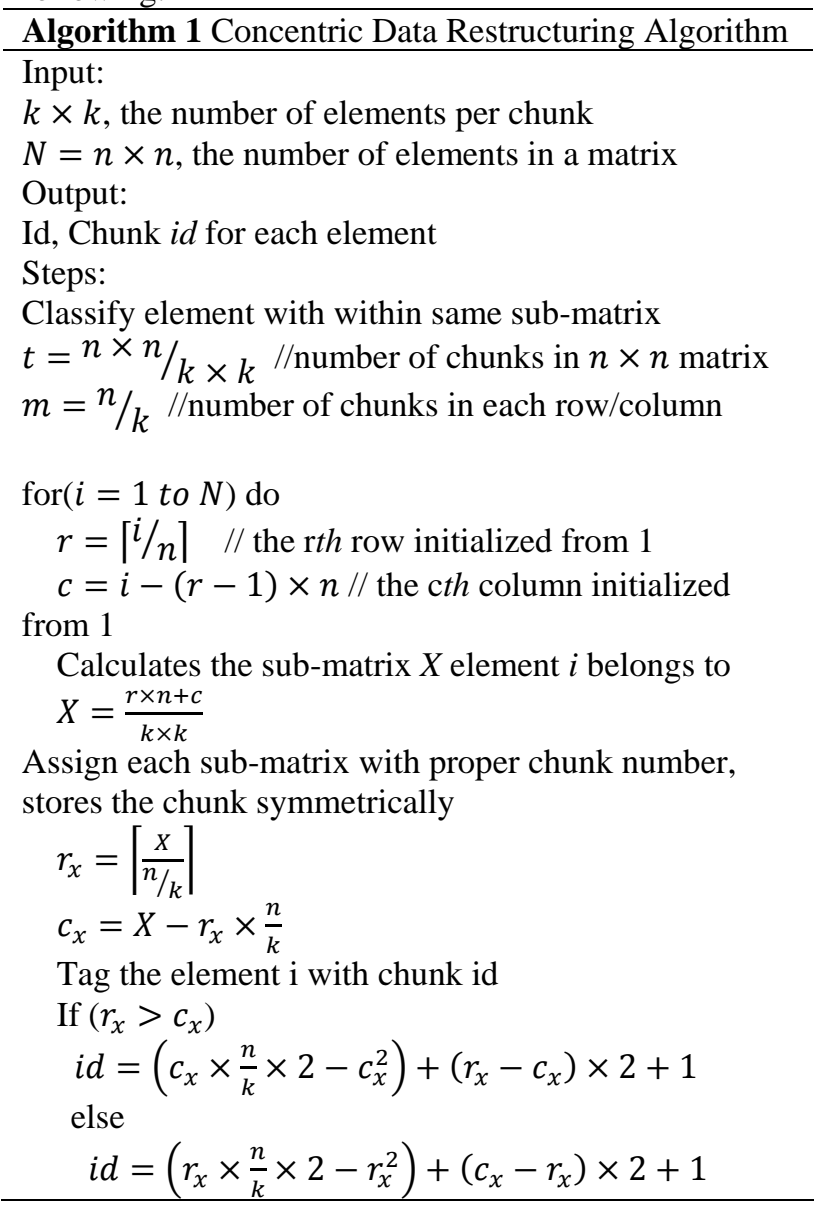

\subsection{Mathematical Analysis}

We compare the average performance between concentric data layout and continuous data layout based on the following assumptions.

First, the data set can be accessed by different access patterns.

Second, the access patterns are row based, column based or group based.

Third, the possibility for each access pattern is equal.

Table 1. Performance comparison between continues and concentric data layout

\begin{tabular}{|c|c|c|c|}
\hline Access pattern & Row based & Column based & Group based \\
\hline Continuous & $1 / 2(n+n \times n)$ & $n \times n$ & $n \times n$ \\
\hline Concentric & $1 / 2(2 n-1+$ & $1 / 2(2 n-1+$ & $1 / 2(2 n-$ \\
& $n \times n)$ & $n \times n)$ & $1+n \times n)$ \\
\hline
\end{tabular}

Suppose the matrix file with the size of $n \times n$ and the size of the chunk is $k \times k$. For continuous data layout, each row contains $\frac{n}{k \times k}$ chunks and there are $\frac{n \times n}{k \times k}$ chunks in total. Then row based access will require $\frac{n+n \times n}{2 k \times k}$ chunks on average, column based access and group access pattern will require $\frac{n \times n}{k \times k}$ chunks each time. The average number of chunks accessed $N_{\text {continuous }}=\frac{n+n \times n}{2 k \times k} \times P_{\text {row }}+\frac{n \times n}{k \times k} \times P_{\text {col }}+\frac{n \times n}{k \times k} \times$ $P_{\text {group }}$. For concentric data layout, each row and column contain exactly $\frac{n}{k}$ chunks and the average number of chunks accessed is

$N_{\text {concentric }}=\frac{1}{2}\left(\frac{2 n}{k}+1+\frac{N \times N}{k \times K}\right) \times P_{\text {row }}+\frac{1}{2}\left(\frac{2 n}{k}+1+\right.$ $\left.\frac{N \times N}{k \times K}\right) \times P_{\text {column }}+\frac{1}{2}\left(\frac{2 n}{k}+1+\frac{N \times N}{k \times K}\right) \times P_{\text {group }}$.

The comparison between two data layout is $P_{\text {concentric }} / P_{\text {continuous }}=\frac{2 n}{\sqrt{k}} / n \times(3 n+1) /_{4 k}=\frac{8 \sqrt{k}}{3 n}$.

As $n$ is larger than $k$ in Hadoop file system, concentric data layout results less chunk access and relieves the data overhead.

\section{Performance Evaluation and Analysis}

In this section we evaluate the performance of concentric algorithm against the continuous access pattern. Because most of the HPC analytics applications with group access patterns still need to be developed, there are no established benchmarks available to test our design. We carry out a prototype implementation with group data layout and matrix data 
layout on Hadoop File System based on the previously discussed data layout optimization algorithm. We analyze the experiment result in following sections and demonstrate the concentric data layout reduces the amount of data accessed, relieves the data overhead, solves the small $\mathrm{I} / \mathrm{O}$ problem and improves the working efficiency.

\subsection{Experimental Setup}

In our experiment, we access to a 17 node cluster with Hadoop 0.20 installed on it. In our setup, the cluster's master node is used as the NameNode and JobTracker, whereas the 16 slave nodes are configured to be the DataNodes and TaskTrackers. In this experiment, we were mainly concerned number of data retrieved and number of map task processed.

During experiment, we write a MapReduce program to process the data set with group data access property by two different data layouts, the original continuous data layout and the optimized concentric data layout. In the map phase each process reads contiguous chunks and marks all the required data. In the reduce phase, all the data required by a single process are combined together. Then we analyze the performance in aspects in executing time, amount of accessing data, useful data efficiency (defined as the ratio of data needed to the amount of data read in MapReduce) and number of map tasks.

\subsection{Experimental Analysis}

We perform a series of tests on the Hadoop cluster to compare the performance on different layout strategies. We write the MapReduce program to process two dimensional files with the size of $1 \mathrm{~GB}$, 4GB, 16GB and 64GB by using different data layout respectively. These files are originally stored in Hadoop file system with continuous data layout, and then they are processed by concentric algorithm and stored in Hadoop file system. In our experiment, each task process one chunk with the default size of 64MB.

First, the experiments are conducted to show the improvement on the execution time of the applications using MapReduce program to access data between concentric data layout and continuous data layout. In the experiments, we have the application to access the data which group located in different position, different row and column in the matrix. Figure 4 shows the performance on the execution time with accessing data in different group (row and column) by using concentric data layout and continuous data layout.

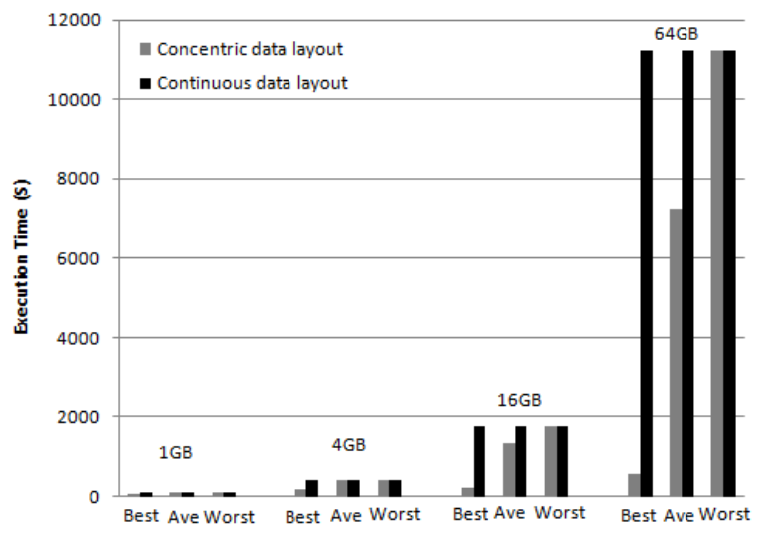

Figure 4. Executing time comparison

From the Figure 4, we can see that when accessing the data located in the last row and column the executing time with concentric data layout and continuous data layout are similar. Take 16GB file for example, the accessing time are both around 1,720s. However, when accessing the data in the beginning of the matrix, take the first row and column for example, we can see the huge saving of time with concentric data layout. The execution time to process 16GB file with concentric data layout is $217 \mathrm{~s}$ while the time to process the same file with the continuous data layout is 1,752s. This improvement can be observed in other files with 1GB, 4GB and 64GB file size. We also let the applications to require data in the middle of matrix. In this case, the execution time with concentric data layout is still better preformed than the execution time with continuous data layout. From the Figure 4, we can get the conclusion that the MapReduce program execution time with concentric data layout is better than that with continuous data layout. This is consistent with our model and analysis in Section 3. In best case which the data set required are located at first row and column, the MapReduce program deal with concentric data layout data set just need to retrieve the few chunks to get all necessary data. However, the MapReduce program which deals with continuous data layout data set has to retrieve almost all the chunks to get all required data because the data are stored in a sequential way in Hadoop File System. In the worst case which the application need to access the data in the last row and column in the matrix, both MapReduce programs have to retrieve all the chunks to get the required data set. However, the reasons are different. For MapReduce program with concentric data layout, all the chunks have to be retrieved because the required data is located in the bottom chunks and the MapReduce program has to process the chunks sequentially from the beginning. For MapReduce program with continuous data layout, the program has to process all the chunks because the data required is 
located in different chunks and the data in last row is stored in the last chunk. Based on the result from Figure 4 and the above analysis, we can see that concentric data layout has better performance than continuous data layout on I/O system performance with execution time.

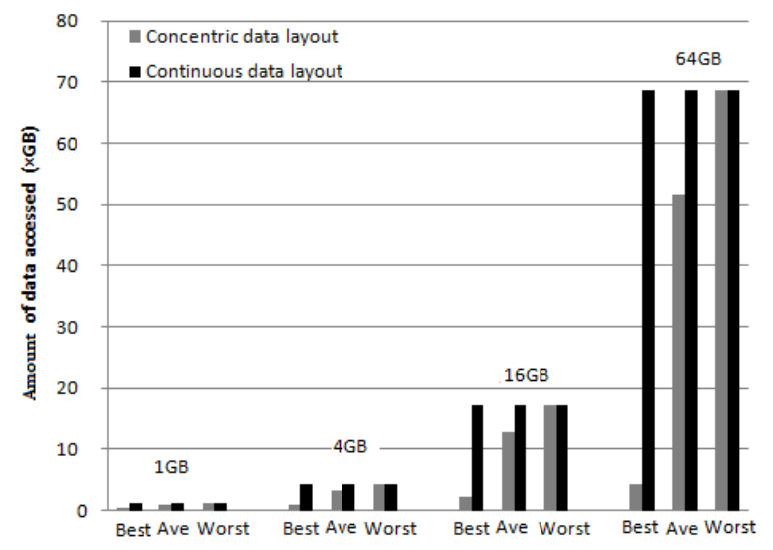

Figure 5. Amount of data accessed

Second, the experiments compare the amount of data accessed by concentric data layout and continuous data layout. From Figure 5, it clear to see the concentric data layout results in less data which accessed by the MapReduce program than the MapReduce program with continuous data layout. Take file with 1GB for example, the average data set retrieved by MapReduce program with concentric data layout is $704 \mathrm{MB}$ while the data set retrieved by MapReduce program with continuous data layout is $1 \mathrm{~GB}$. As the file gets larger, this advantage becomes more evidence. In 64GB file, the average data size of 48GB is accessed with concentric data layout while the average data size of 64GB is accessed with continuous data layout. The saving is up to $25 \%$. The difference caused by the fact that in order to access all require data, MapReduce program needs to retrieve the chunks from the beginning. Concentric data layout reconstructs the data and stores the data with same group in same or close chunks. Therefore, compares with continuous data layout, concentric data layout makes MapReduce program retrieves fewer chunks and hence accesses less data.

Meanwhile, we compare the useful data efficiency (data efficiency in brief for the rest) with two different data layouts. Take 16GB file for example, from Figure 6 , we can see that, compared with continuous data layout, the concentric data layout improves the data efficiency. In the best case which the application requires data in first row and column, the data efficiency increased from $6.25 \%$ with continuous data layout to $51.6 \%$ with concentric data layout.

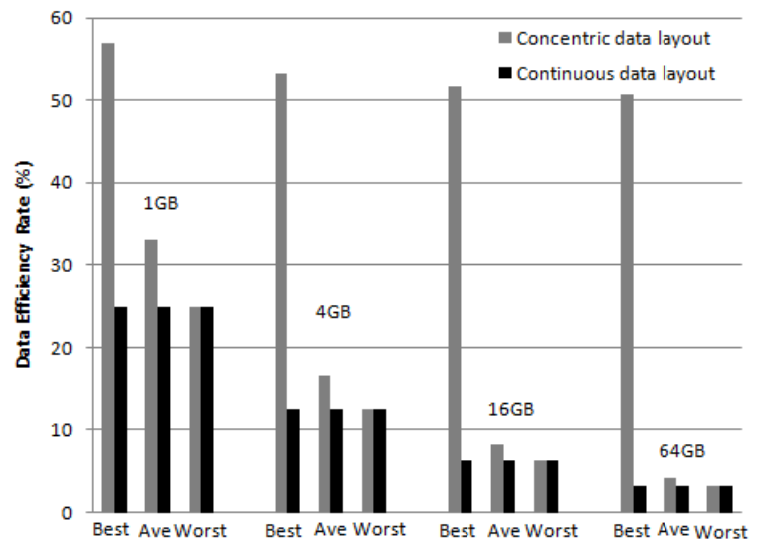

Figure 6. Data efficiency comparison

Though in worst case which the application needs to retrieve the whole file, the data efficiencies are close with two data layout patterns, the average data efficiency is improved from $6.25 \%$ with continuous data layout to $8.33 \%$ with concentric data layout. The experiment results are consistent with our theoretical analysis. As we have analysis, concentric data layout makes MapReduce program retrieve less chunks, which avoids to read unnecessary data and relieve the data overhead. Since both MapReduce programs require same amount of data from file, the fewer chunks retrieved by the program, the higher efficiency the data layout provides.

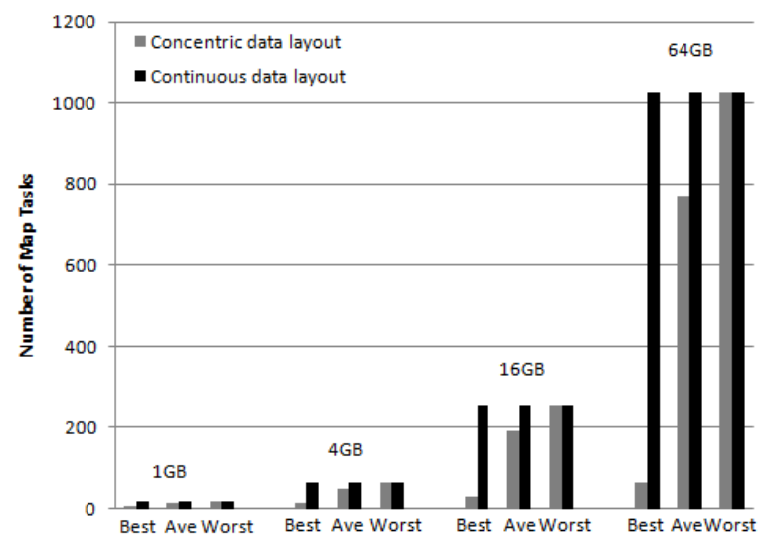

Figure 7. Number of Map Tasks

Third, we compare the number of map tasks the MapReduce program has with concentric data layout and continuous data layout respectively. From the task, it is clear to see that concentric data layout has reduced the number of map task dramatically. From the Figure 6 we can see accessing data in concentric data layout with 16GB file needs to have 192map tasks on average, while accessing data in continuous data layout with 16GB file require 256 map tasks. The improvement is caused by the fact that concentric data 
layout stores the data within same group into close chunks. When application requires group data, it needs to retrieve fewer chunks to read all required data. As we have mentioned, in our experiment each map task processes one chunk with the default chunk size of 64MB. Therefore, the fewer chunks the application retrieved, the less map tasks it required. Compare with continuous data layout, concentric data layout makes MapReduce program accesses less chunks and hence require less map tasks. From the experiment result, we can draw the conclusion that the concentric data layout relieves task scheduling problem.

\section{Related Work}

Many approaches have been adopted to relieve the small I/O problem in HPC application, especially for applications using MPI/MPI_IO. Data sieving[2] is an optimization technique to deal with small I/O problem. According to data sieving algorithm, instead of accessing each contiguous portion of the data separately, a single contiguous chunk of data starting from the first requested byte up to the last requested byte is read into a temporary buffer in memory. The advantage of this algorithm is that data is always accessed in large chunks. However, the limitation of this simple algorithm is obvious. The data sieving requires the temporary buffer into which data is first read must be as large as the total number of chunk, which generates excessive amount of unnecessary data. Collective $\mathrm{I} / \mathrm{O}[2]$ also allows process to read a contiguous chunk of data but then using MPI framework, it redistributes the data among multiple processes as required by them. Besides, applying collective I/O with two-phase implementation in large scale system will result in communication overhead among processes. PLFS[3] is another approaches for small I/O problem. PLFS is a file system which mounted on the top of an existing parallel file system and re-maps an applications' write access pattern to be optimized for the under-laying file system. DFS[4] provides striping mechanisms that divides a file into small pieces and distributed them across multiple storage devices for parallel data access. Our work is different from the above mentioned approaches. In our work, we reconstruct the data layout and processes do not need to communicate with others due to the data reorganization. Our work successfully maintains the shared-noting architecture for scalability. DPFS[5] proposed a multi-dimension data layout to process matrix data set. It takes the data relationship in the matrix data set into consideration and improves the data access efficiency. However, the multi-dimension data layout just focus on row/column based data access. In our work, the concentric data layout can work with more complicated access requirement like group data access pattern.

\section{Conclusion}

In this paper we present a concentric data layout algorithm to support data analytics applications using matrix data structures. Concentric data layout is an optimization strategy which works well with various data access patterns among a matrix-structured data set. It is a hierarchical data layout which maintains the dimensional property in a multidimensional way. In concentric data layout, instead of storing the data into chunks continuously, data located within the same submatrix is stored into the same chunk, and then chunks are stored into Hadoop file system symmetrically. The concentric data layout is able to significantly boost the I/O performance for data analytics programs by matching with their mixed row-based and columnbased access patterns. Our experiments on a revised Hadoop prototype show that, given a concentric layout, a MapReduce program accesses fewer chunks when reading a group of data in a matrix file compared to current continuous layout in Hadoop file system, and thereby significantly improve the I/O read performance for matrix specific data analysis applications.

\section{Acknowledgements}

This work is supported in part by the US National Science Foundation under grants CNS-0646910, CNS0646911, CCF-0621526, CCF-0811413, US Department of Energy Early Career Principal Investigator Award DE-FG02-07ER25747, and National Science Foundation Early Career Award 0953946.

\section{References}

[1] http://hadoop.apache.org/common/docs/current/hdfs_desig n.html.

[2] Rajeev Thakur, William Gropp, Ewing Lusk, Data Sieving and Collective I/O in ROMIO," frontiers, pp.182, The 7th Symposium on the Frontiers of Massively Parallel Computation, 1999.

[3] John Bent, Garth Gibson, Gary Grider, Ben McClelland, Paul Nowoczynski, James Nunez, Milo Polte, and Meghan Wingate. PLFS: A checkpoint filesystem for parallel applications. In Supercomputing, 2009 ACM/IEEE Conference, Nov. 2009.

[4] JH Howard, ML Kazar, SG Menees. Scale and performance in a distributed file system. ACM Transactions on Computer Systems, Volume 6, Issue 1, 1988.

[5] Xiaohui Shen, Alok N, Choudhary. Dpfs: A distributed parallel file system. In ICPP 02: Proceedings of the 2001 
International Conference on Parallel Processing, pages 533544, Washington, DC, USA, 2001.

[6] D. Borthakur. The Hadoop Distributed File System: Architecture and Design. Apache Software Foundation, 2007.

[7] Bryant, R. E. Data-Intensive Supercomputing: The Case for DISC. Tech. Rep. CMU-CS-07-128, Carnegie Mellon University, May 2007.

[8] V. Akcelik, J. Bielak, G. Biros, I. Epanomeritakis, A. Fernandez, O. Ghattas, E. J. Kim, J. Lopez, D. R. O’Hallaron, T. Tu, and J. Urbanic. High resolution forward and inverse earthquake modeling on terasacale computers. In Proceedings of SC2003, November 2003.

[9] G. Mackey, S. Sehrish, J. Lopez, J. Bent, S. Habib, and J. Wang, Introducing mapreduce to high end computing, in Petascale Data Storage Workshop held in conjunction with SC08, 2008 